\title{
FLUORESCENT ANTISERA IN THE DETECTION OF SEROLOGICAL VARIETIES OF TRICHOMONAS VAGINALIS*
}

\author{
BY \\ M. G. McENTEGART, C. S. CHADWICK, AND R. C. NAIRN \\ From the Departments of Bacteriology, Biochemistry, and Pathology, University of Aberdeen
}

In spite of extensive research devoted to all aspects of the problem of trichomoniasis (reviewed by the Société française de gynécologie, 1957), we still know little about the factors which determine the severity of the infection. The clinical reaction to infection with Trichomonas vaginalis varies from the symptomless carrier state to acute vaginitis, and this variation might, in theory, be due to differences in the host response, in the size of the infecting dose, or in the type of the infecting organism. Of these, the last possibility was one that could be investigated in the laboratory. If serotypes do in fact exist, they may help to explain the apparent variation in the infectivity and virulence of the organism. Moreover, the detection of such serological types would be most useful in studying the epidemiology of the infection.

Search for serological variants is handicapped by the lack of a suitable test directly applicable to vaginal discharges. At present, using agglutination methods, all strains must be well established in culture before any test is possible. This is timeconsuming and not all new isolations reach the required level of growth, though recent work shows that successful cultivation, using a modified medium, can now be achieved with a large proportion of strains (Whittington, 1957; Feinberg and Whittington, 1957). Nevertheless, a direct typing method applicable to fixed vaginal smears or to the scanty organisms from an early culture would be invaluable. We felt that the method of Coons (reviewed comprehensively by Coons, 1956), using the specific staining ability of antibody labelled with fluorescein, might be suited to the study of this problem. Such an

* Received for publication October 25, 1957.

tPaper read to the M.S.S.V.D. in London on October 25, 1957. antibody has the property of coating the corresponding antigen, which can then be identified by ultra-violet fluorescence microscopy. The equipment we used for this purpose was the Reichert fluorescence microscope with a high pressure mercury vapour lamp as the light source; a full description of the apparatus with details of the photomicrography has been given elsewhere (Nairn, Chadwick, and McEntegart, 1957).

Anti-Trichomonas vaginalis serum was prepared in rabbits as previously described (McEntegart, 1956), and in our initial experiments the globulin fraction of the serum was separated and conjugated to fluorescein isocyanate, the conjugate being subsequently purified by repeated ethanol fractionation and absorption twice with dried liver powder. We found that less-purified preparations gave excessive non-specific staining, evidenced in the early experiments with $T$. vaginalis by a failure of unconjugated immune serum to inhibit the attachment of subsequently applied conjugated immune serum. This reaction, usually spoken of as "blocking", is an essential proof that the staining observed is due to the specific localization of fluorescein-conjugated antibody. The final conjugated serum was applied to acetone-fixed smears of $T$. vaginalis from pure culture and gave somewhat less brilliant staining than the crude conjugate, but this was more than compensated for by the specificity of the reaction, which could now be blocked.

Having established that the homologous strain of $T$. vaginalis could be stained specifically, we next applied the method to the examination of acetonefixed smears of vaginal discharge. Material from known positive cases, and from negative cases as controls was obtained locally, and also from 
Liverpool, London, and Dublin. The technique is more difficult to apply to vaginal smears than to pure cultures; there is a confusing bright autofluorescence of some of the cellular and other elements in the discharge and also troublesome non-specific staining of leucocytes. We have not yet been able to overcome this difficulty, but extension of the purification methods offers some hope of a solution. Although the method is unsuitable for the identification of $T$. vaginalis in fixed smears, being no better than staining by Giemsa's method, it clearly has a definite value in research.

Before trying to detect what are probably rather subtle antigenic differences between strains of $T$. vaginalis, we carried out a simple test (Fig. 1) to see if the method could distinguish between $T$. vaginalis and $T$. foetus (var. Belfast). The deposited protozoa from young cultures of $T$. vaginalis and $T$. foetus were used to prepare three films on one microscope slide. The first consisted of $T$. vaginalis only, the third of T. foetus only, and the second of a mixture of approximately equal numbers of each. After acetone fixation, the three films were covered with one pool of conjugated anti- $T$. vaginalis globulin, and in this way ali three films were treated with the same serum for the same time. After washing in buffer, the films were mounted in glycerol-buffer and examined by fluorescence microscopy. The resulting appearance of the mixed

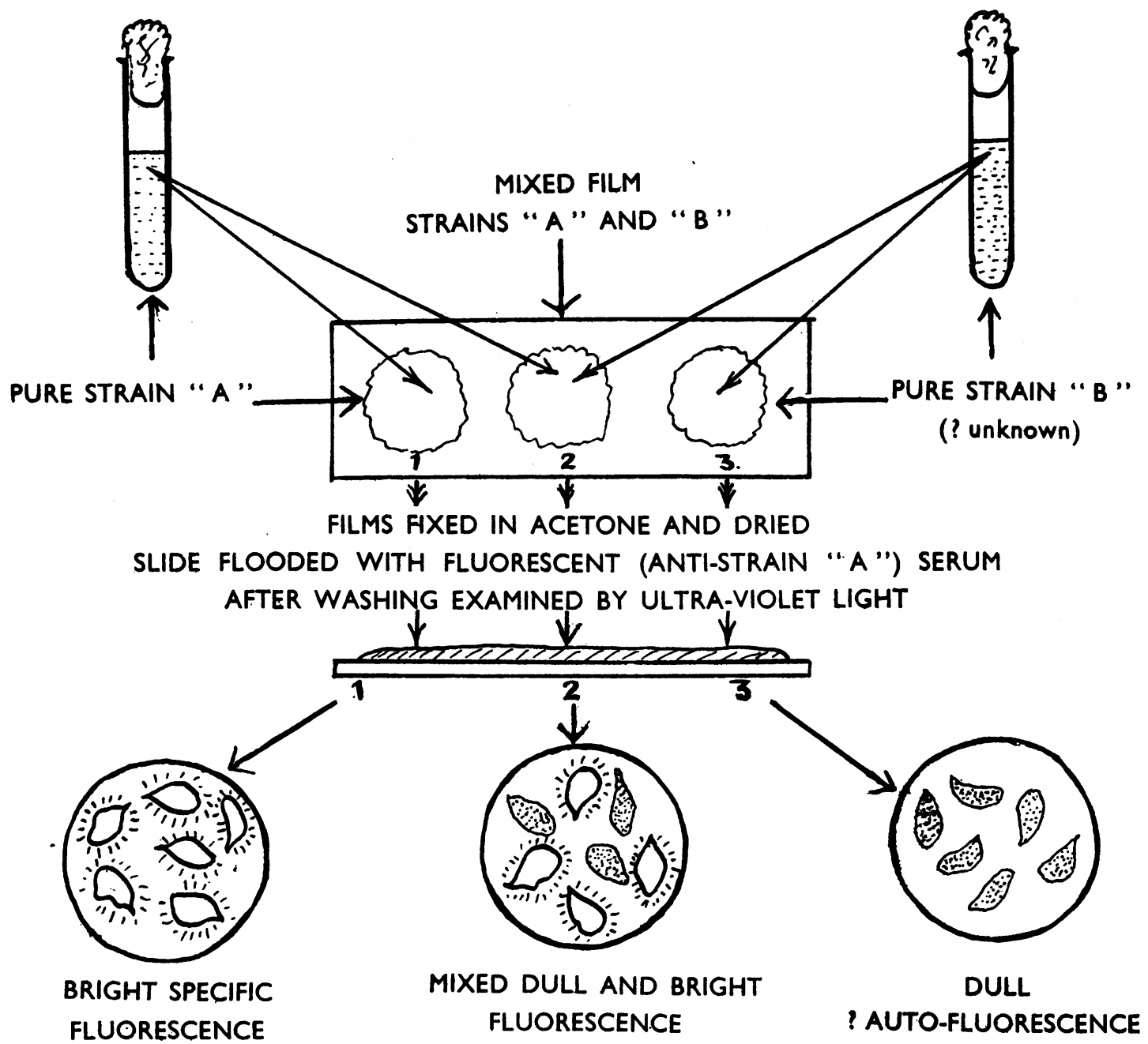

Fig. 1.-Use of a single fluorescent anti-Trichomonas serum as a possible method of demonstrating serologically distinct strains. 
film of the two protozoa is shown in Fig. 2. Although this result was regularly repeatable, we felt it did not alone provide sufficient evidence for the specificity of the reaction. Accordingly we carried out a like procedure using conjugated anti$T$. foetus (var. Belfast) serum, and this as predicted led to a reversal of the bright and dull fluorescence in the mixed films.

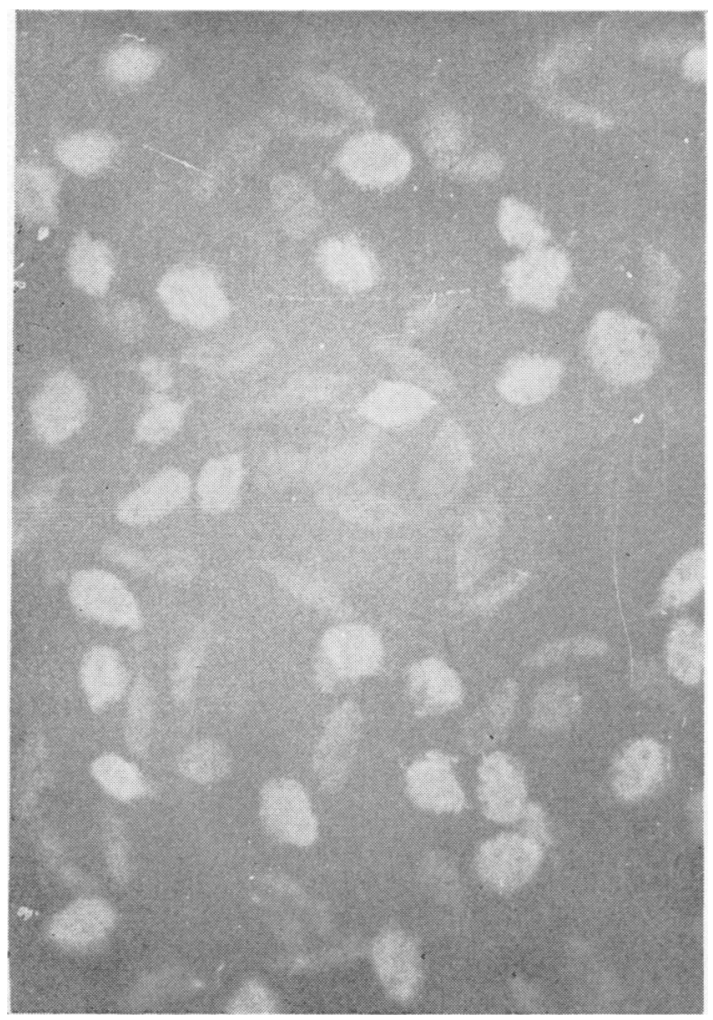

Fig. 2.-Appearance of mixed film of protozoa ( $T$. vaginalis and T. foetus).

Having thus established that the known wide antigenic differences between $T$. vaginalis and $T$. foetus could be demonstrated by means of fluorescent antibody, we repeated the same "three spot test" with a culture of $T$. vaginalis which had been isolated by Dr. Lanceley and which he suggested might be serologically distinct from our original Liverpool stain. We have not yet had time to prepare an antiserum to this strain so that all we can say is that Lanceley's "Liverpool 52" strain certainly fluoresces less brightly than the original Liverpool strain, and this suggests that we may well be dealing with a serologically distinct variety of $T$. vaginalis. Proof of this view will depend upon the reactions observed with the conjugated "anti52" serum with, if necessary, cross-absorption after conjugation. If Lanceley's strain does prove to be a distinct variety, it would suggest that the fluorescent method may be a quick and convenient way of investigating the distribution of this and other possible serotypes.

Whatever the immediate value of these observations in the study of trichomoniasis there is a more general interest in the application of the method to the study of other protozoological problems. The technique has been applied by others to the study of Entamoeba histolytica, toxoplasma, and paramecium (Goldman, 1953, 1954, 1957; Beale and Kacser, 1957), but there are undoubtedly special difficulties to be overcome before it can be generally used in protozoology or protozoan pathology. Nevertheless it is our experience that the labour of the preliminary groundwork is amply repaid by the fascination of an experimental method which offers a new approach to the immunological problems of protozoa.

We wish to thank Dr. Muriel Robertson for the culture of $T$. foetus and all our colleagues who helped us by providing clinical specimens. This research programme has been supported by a grant from the Scottish Hospital Endowments Research Trust.

\section{REFERENCES}

Beale. G. H., and Kacser, H. (1957). J. gen. Microbiol., 17, 68. Coons, A. H. (1956). Int. Rev. Cytol., 5, 1.

Feinberg, J. G., and Whittington, M. J. (1957). J. clin. Path., 10, Goldman.

Goldman, M. (1953). Amer. J. Hyg., 58, 319.

(1954). Ibid., 59, 318.

(1957). J.exp. Med., 105, 549.

McEntegart, M. G. (1956). J. Path. Bact., 71, 111.

Nairn, R. C., Chadwick, C. S., and McEntegart, M. G. (1957). Ibid., (in press).

Société française de gynécologie (1957). "Les infestations à trichomonas", I Symp. Europ. Masson, Paris.

Whittington, M. J. (1957). Brit. J. vener. Dis., 33, 80. 